\title{
Bridge collé en zircone : à propos d'un cas
}

\section{Zirconia Resin-bonded bridge: a case report}

\section{MOTS-CLEFS :}

- Bridge collé, bridge maryland, tout-céramique, céramique zircone

\section{KEYWORDS:}

- Resin-bonded bridge, maryland bridge, all-ceramic, zirconia

$\operatorname{AOS} n^{\circ} 283-2017$

\section{Résumé}

Devant un cas d'édentement unitaire, si les solutions implanto-portées représentent souvent une thérapeutique convoitée, dans certaines situations, les bridges collés constituent une alternative de choix répondant aux impératifs fonctionnels, esthétiques et biologiques. La majorité des bridges collés sont en alliage métallique précieux ou non-précieux. Une alternative à l'utilisation du métal est la réalisation de bridges collés "tout céramique ». Cet article propose une réflexion au sujet de l'utilisation d'un bridge collé tout-céramique et qui sera illustre ò travers un cas de remplacement d'une première prémolaire maxillaire.
Abstract

In single tooth missing cases, dental implant represents the most coveted solution. However, resin-bonded bridge can be, in some situations, an option of choice including functional, biological and esthetic requirements. Mostlty the resin-bonded bridges are made of metalic retainers. An alternative excluding the use of metal, is all-ceramic resin-bonded bridge.

This paper is a reflexion about the use of all ceramic resin-bonded bridges and is illustrated with a case which describes the replacement of a missing first premolar.

\begin{tabular}{|l|l|}
$\begin{array}{l}\text { Hicham SOUALHI, Professeur agrégé en prothèse fixée - Département } \\
\text { de prothèse fixée - Faculté de médecine dentaire, Université Mohammed V, } \\
\text { Rabat Maroc. } \\
\text { E-mail : soualhihicham @yahoo.fr }\end{array}$ \\
\hline IIV
\end{tabular}

\section{INTRODUCTION}

Actuellement la médecine dentaire restauratrice se traduisent par un changement radical des concepts : plus de respect des tissus sains et des impératifs esthétiques, mais également une préoccupation constante de limiter la complexité des traitements prothétiques. Ainsi, bien que les situations d'édentement unitaire soient extrêmement fréquentes le choix final raisonné d'une seule option de traitement n'est pas toujours facile. Cette situation offre l'un des éventails thérapeutiques les plus larges. Lorsque la mise en place d'un implant est impossible pour des raisons financières, sociales ou médicales, la possibilité de réaliser une restauration adhésive (bridge collé) doit être systématiquement envisagée (1).
Cette modalité thérapeutique, longtemps considérée comme une solution provisoire de longue durée, a fait la preuve de sa fiabilité $(2,3,4)$. Cette technique est intéressante, non seulement par l'économie de tissu qu'elle garantit, mais également par sa sécurité inégalée sur le plan biologique.

Depuis l'introduction des bridges collés perforés par Rochette (5) en 1973, les concepts thérapeutiques et les matériaux et technologies à disposition dans ce domaine ont connu une constante évolution. C'était le premier type de prothèse fixée non invasif car les dents piliers ne subissaient aucune préparation préalable. Le champ d'application était limité au remplacement d'une dent dans les secteurs antérieurs. Cette technique a connu, après, un réel engouement au début des années 80 avec les propositions de l'école de Maryland 
(6). Ces propositions impliquaient des préparations à minima fondées sur les concepts de stabilisation des moyens d'ancrage de prothèse amovible partielle, et le traitement de surface des infrastructures métalliques par mordançage électrolytique. L'indication des bridges collés s'étendait au remplacement des dents postérieures.

À l'origine de bridges collés étaient fabriqués en alliages nobles ou de $\mathrm{Ni}-\mathrm{Cr}$, les alliages chrome-cobalt représentent actuellement le matériau préconisé. L'avantage de ces alliages se fonde principalement sur leur module d'élasticité élevé, ce qui permet la réalisation d'armatures plus fines, tout en assurant une bonne résistance mécanique. De même, ils présentent une bonne adhérence au composite de collage (7) et permettent d'assurer une liaison céramo-métallique de bonne qualité (8).

Cependant, parallèlement aux évolutions dans le domaine de la prothèse fixe conventionnelle, des facteurs comme l'amélioration de l'esthétique (réduction/ élimination de l'aspect grisâtre de l'armature) et le désir de travaux exempts de métal et d'une biocompatibilité maximale associes aux progrès dans le domaine du « tout-céramique » ont abouti à proposer les bridges collés tout céramiques.

\section{CAS CLINIQUE}

Il s'agit d'un patient âgé de 24 ans qui consulte dans le but de remplacer la 14 extraite pour raison carieuse. Il est en bon état de santé générale. Le patient nous fait

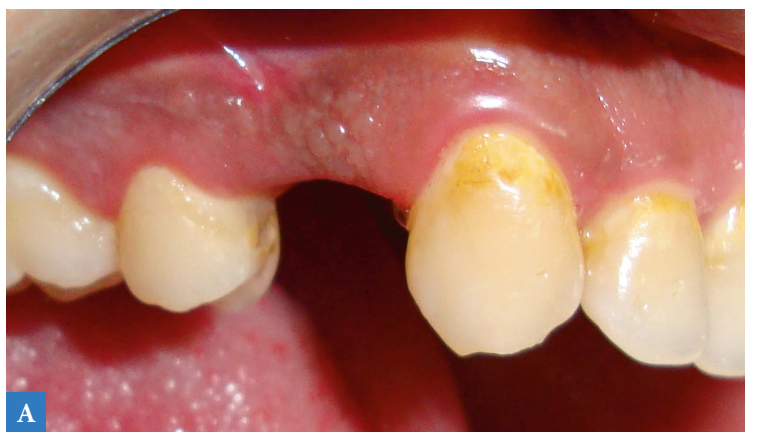

part de son refus d'une solution amovible tout en insistant sur le désir d'une restauration esthétique durable. L'examen clinique objective un bon niveau d'hygiène orale. L'examen des dents bordantes l'édentement révèle que la 13 est sain, cependant la 15 présente une petite carie mesiale. Ces deux dents sont en normopostion. Leurs indices mésio-distaux (LE HUCHE) sont peu élevés et leurs hauteurs coronaires satisfaisantes surtout favorables à la mise en place de connexions de diamètre important (Fig 1a, 1b). La zone édentée est en cours de cicatrisation suite à l'extraction récente de la 14. La longueur de l'édentement coïncide avec la mise en place d'une première prémolaire maxillaire. L'occlusion d'intercuspidation maximale est stable. En propulsion, on observe un guide incisif efficient. En diduction droite et gauche on constate une fonction canine efficace (Fig 2a, 2b).

Quatre possibilités thérapeutiques s'offrent à nous compenser cet édentement unitaire : prothèse amovible partielle, prothèse implanto-portée, bridge collé et un bridge conventionnel. La solution amovible est d'emblé rejetée compte tenu des doléances du patient. La solution implantaire est écartée pour des raisons financières. Le délabrement excessif des dents piliers lors de la réalisations d'un bridge conventionnel nous incite a privilégier une solution plus conservatrice en l'occurrence un bridge collé.

Le patient a exprimé le souhait d'éviter la présence d'alliages métalliques. Nous avons opte pour un bridge collé en céramique en zircone de la 13 a 15 tout en informant notre patient du faible recul clinique de ce type de restauration.

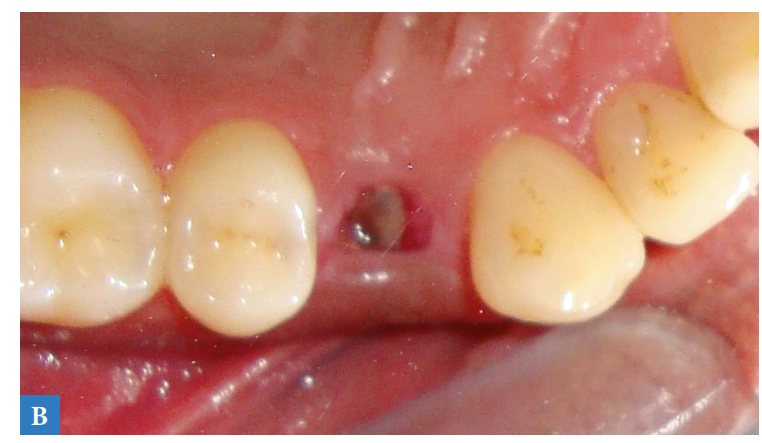

$\triangle$ Fig. 1 : État initial, a) vue vestibulaire b) vue occlusale > Absence de la 14, la 13 est saine la 15 présente une petite carie mesiale.

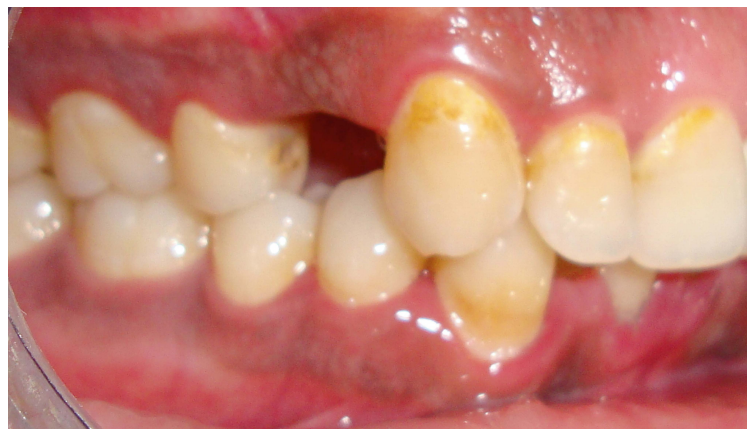

$\triangle$ Fig. 2a : Occlusion en OIM stable.

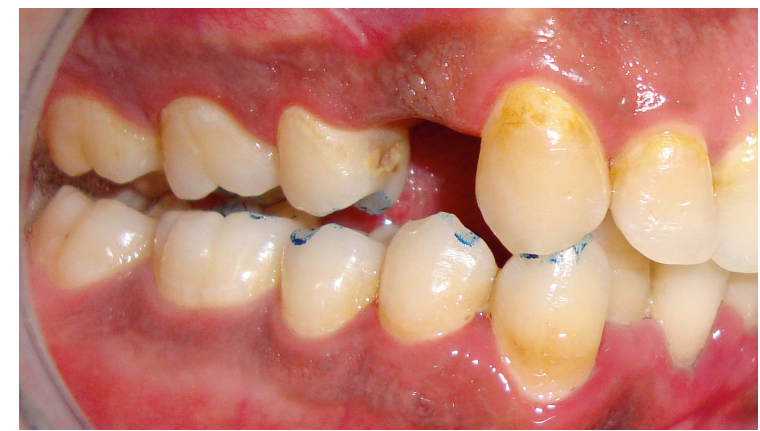

$\triangle$ Fig. 2b : Guidage canin avec absence désocclusion postérieure. 
La préparation a comme objectif de :

I Ménager un volume suffisant et homogène pour le matériau céramique assurant des qualités mécaniques et esthétique optimales.

I Permettre une insertion précise de la restauration (Fig. 3a, 3b).

Au niveau de la prémolaire, en englobant la carie, la cavité a les caractéristiques d'une préparation pour un inlay céramique :

I une cavité occlusale de profondeur de $2 \mathrm{~mm}$ et de largeur de $2 \mathrm{~mm}$;

$\checkmark$ une cavité proximale a limite supra-gingivale ;

$\checkmark$ les angles doivent être arrondis, il faut éviter les angles vifs afin de prévenir les tensions internes et faciliter l'insertion des éléments prothétiques ;

$\checkmark$ une dépouille $10^{\circ}$.

Au niveau de canine :

$\checkmark$ une limite cervicale palatine supra-gingivale en forme d'un petit congé de $10 / 10 \mathrm{~mm}$;

I la préparation s'étend sur le cingulum a $2 \mathrm{~mm}$ du bord libre ;

$\checkmark$ préparation cungulaire en forme de cuvette d'une profondeur de $2 \mathrm{~mm}$;

I extension proximale a l'aplomb du point de contact ; $\checkmark$ une dépouille interne nécessaire a l'insertion du bridge.

L'empreinte est réalisée par technique de double mélange avec un silicone réticulant par addition (Président, 3M${ }^{\circledast}$ ) (Fig 4).

Dans le laboratoire, Après numérisation du maitre modèle, l'armature a été élaborée à partir de bloc de zircone (Y-TZP) (Ips e.maxZirCad, Ivoclar Vivadent) en utilisant pour le fraisage le Cerec Inlab (cerec, Sirona Dental company). Des fraiseuses contrôlées numériquement par ordinateur ont été utilisées à l'usine pour façonner un bloc de zirconium optimisé. L'émaillage de l'armature est réalisé avec une céramique fluoro-apatite pressé.

Une phase d'essayage est indispensable, elle permet de valider notre bridge aussi bien à l'état d'armature (Fig. 5a, 5b, 5c) qu'après le montage du la céramique

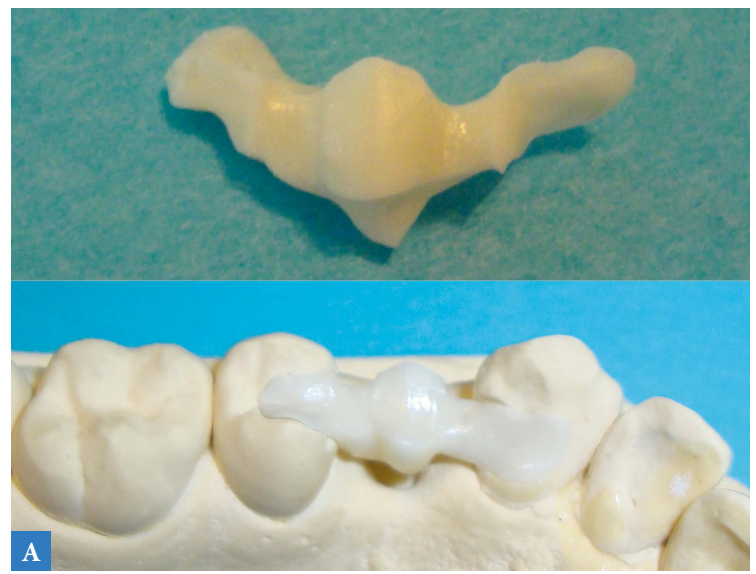

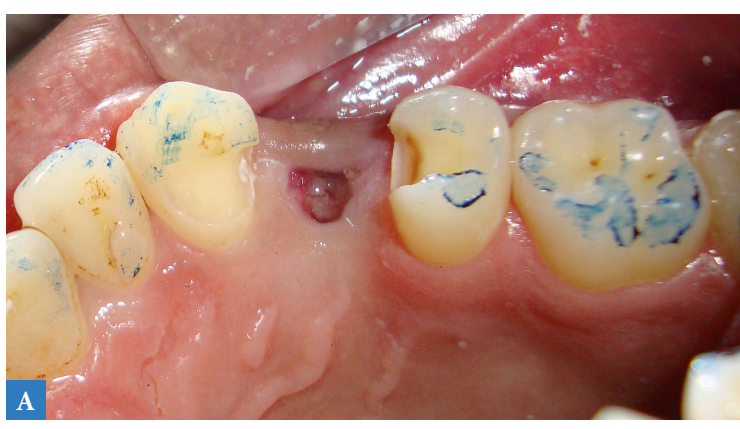

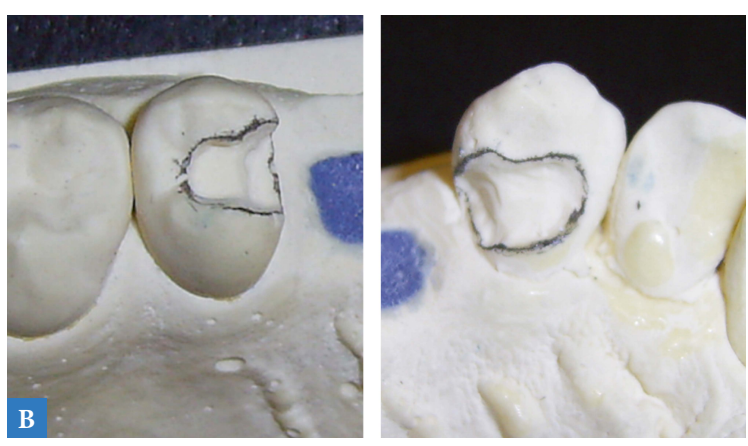

$\triangle$ Fig. 3: Préparation de la 13 et 15.

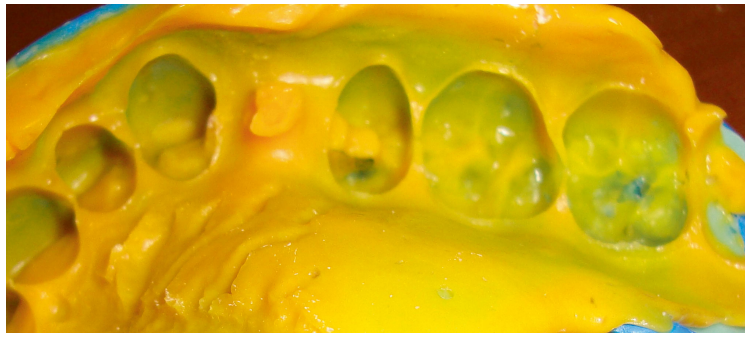

$\triangle$ Fig. 4 : Empreinte en double mélange.

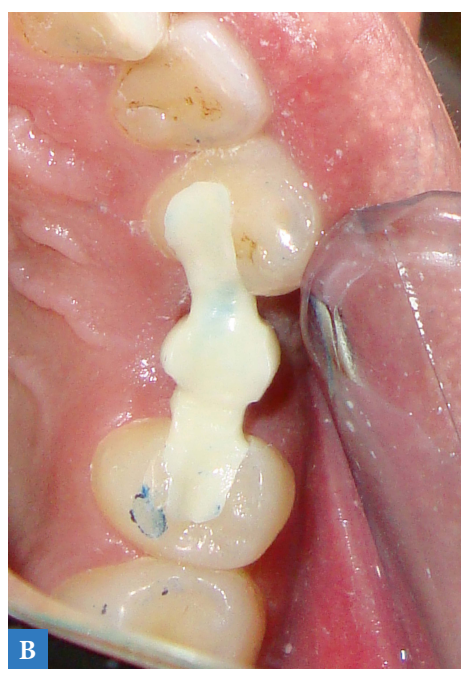

Fig. 5a: Armature

en zircone.

b : vue vestibulaire

de l'essayage de l'armature.

c: vue occlusale de l'essayage de l'armature.

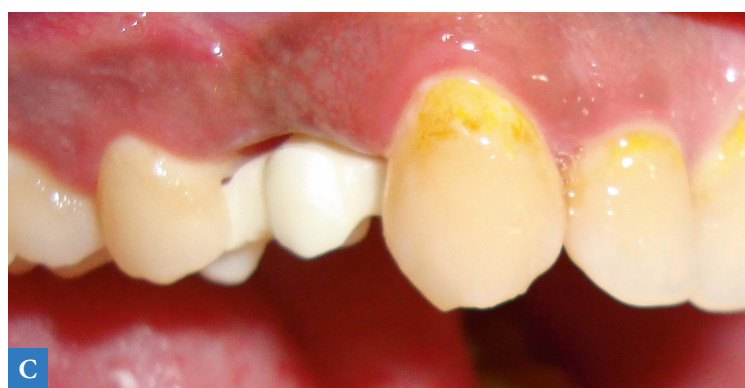


cosmétique (Fig. 6a, 6b, 6c). Au cours de cette phase, il peut être nécessaire d'ajuster des zones de frictions, mais il faut éviter d'essayer de régler l'occlusion à ce stade.

Pour le collage, les céramiques renforcées à la zircone, le traitement à l'acide fluorhydrique est inefficace et ne permet pas de créer à la surface de telles céramiques un relief propice au collage. L'intrados du bridge doit a été traité tribochimiquement (ROCATEC 3m Espe)

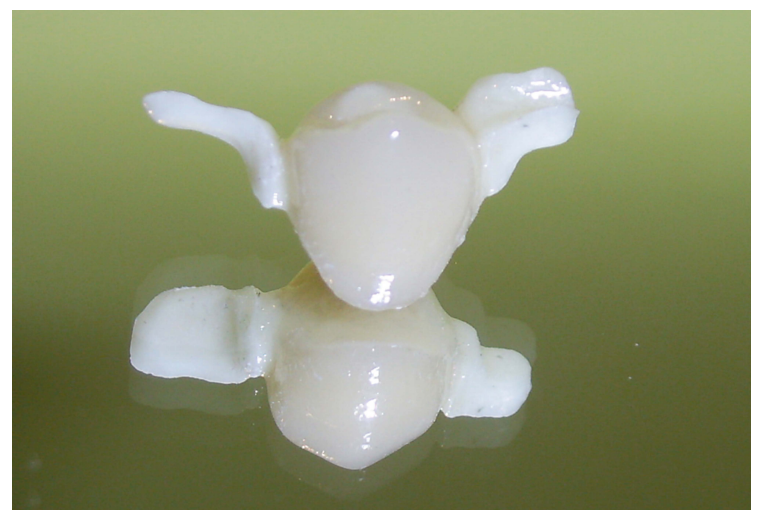

$\triangle$ Fig. 6a : Bridge collé après montage de la céramique cosmétique.
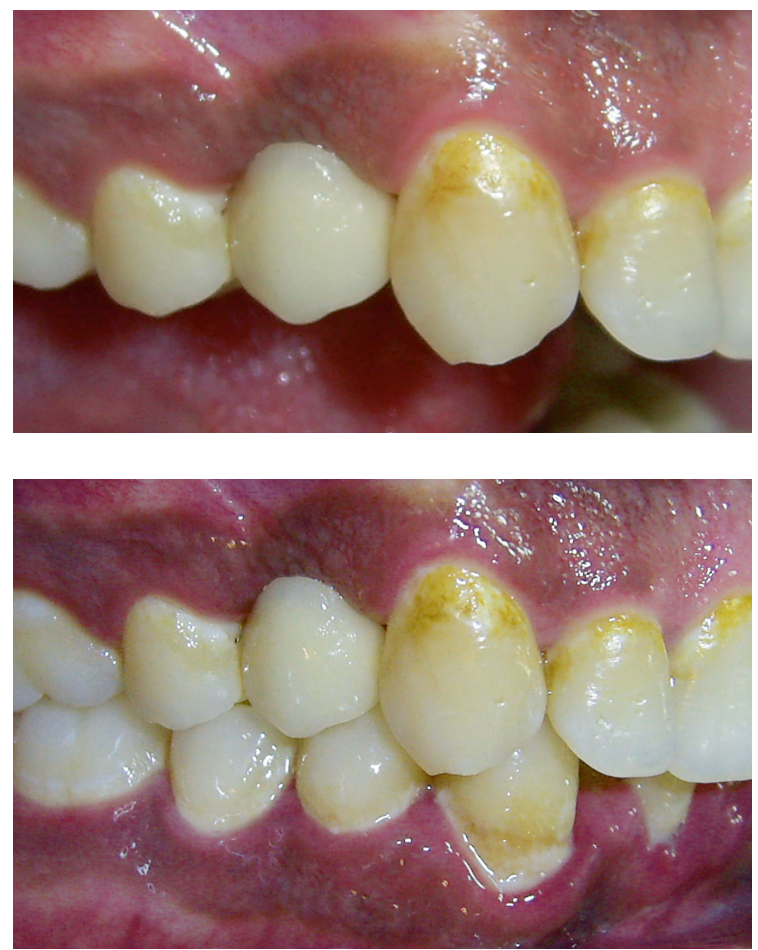

$\triangle$ Fig. 7a : Résultat finale et intégration esthétique du bridge collé. en protégeant la céramique d'émaillage puis silané au Monobond S (Ivoclar Vivadent). Ceci était associé à une composite de collage à potentiel adhésif Panavia $\mathrm{F}$ auto mélangeante.

Après la procédure du collage, on contrôle l'occlusion, et on poli les surfaces retouchées. Le résultat après collage est très satisfaisant, le bridge en zircone n'ayant pas modifié l'aspect des dents adjacentes (Fig. 7a, 7b).

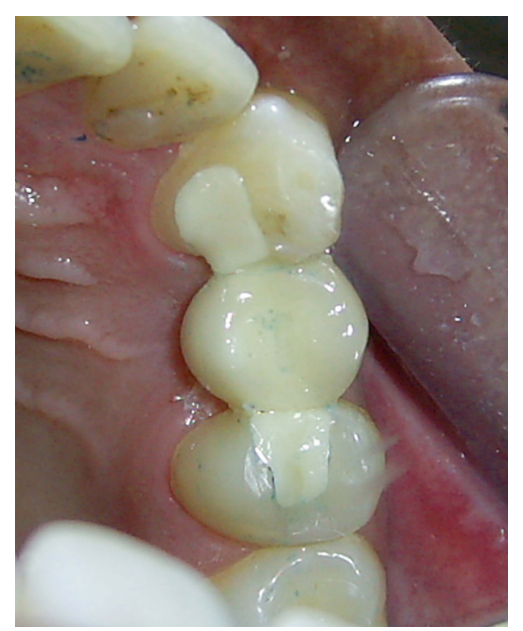

$\triangle$ Fig. 6b : vue vestibulaire de l'essayage du bridge collé. $\lceil$ Fig. 6c : vue vestibulaire de l'essayage du bridge collé.

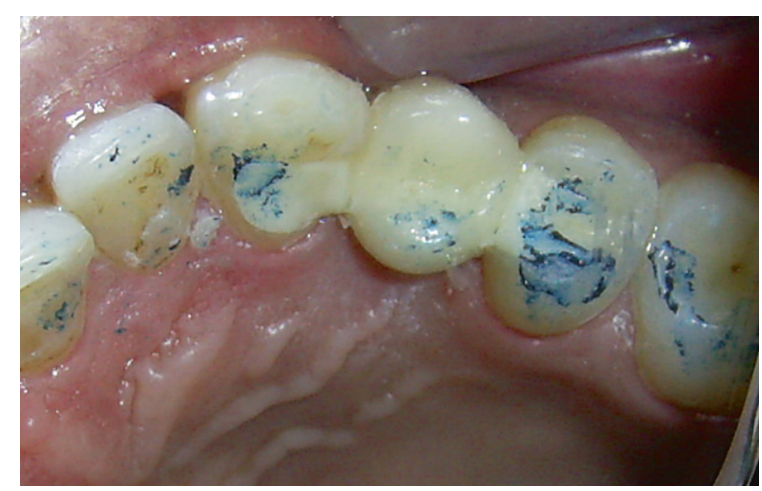

$\triangle$ Fig. 7b : Contrôle de l'occlusion après collage pour s'assurer de l'intégration fonctionnelle du bridge.

\begin{tabular}{|c|c|c|c|}
\hline Système céramique & Flexion MPa & Ténacité MPa m1/2 & Surface de connexion \\
\hline IPS Empress $^{\oplus}$ & 350 & 1,6 & 12 à $20 \mathrm{~mm}^{2}$ \\
\hline Inceram alumina $^{\oplus}$ & 500 & 4,5 & $12 \mathrm{~mm}^{2}$ \\
\hline Inceram zirconia ${ }^{\circledR}$ & 700 & 6,2 & 12 à $20 \mathrm{~mm}^{2}$ \\
\hline Zircone $(\mathbf{Y}-\mathrm{TZP})^{\oplus}$ & 1100 & 9,5 & $9 \mathrm{~mm}^{2}$ \\
\hline Céramo-métallique & & & $6,25 \mathrm{~mm}^{2}$ \\
\hline
\end{tabular}




\section{DISCUSSION}

Différents systèmes de céramique ont été proposés pour la réalisation des bridges collés en céramique. Au début, on a surtout utilisé la céramique d'oxyde d'aluminium infiltré de verre, soit par la technique de la barbotine (Kern et coll. 1991) (9), soit avec recours à la méthode du fraisage par copiage (Kern et Gläser 1997) (10), et plus tard des céramiques infiltrées et renforcées par zircone (Kern et coll. 2005) (11), des vitrocéramiques pressées (Ries et coll. 2006) (12) et des armatures en zircone réalisées par CAD/CAM (Holt \& Drake 2008, Duarte et coll. 2009) $(13,14)$.

Différents matériaux céramique à haute ténacité (par ex : In Ceram ${ }^{\circ}$ alumine/zircon, Vita ; Empress II $^{\circ}$, Ivoclar) $(15,16)$ ont été proposés pour la fabrication de l'armature. Quelques publications décrivent les procédures cliniques spécifiques à chaque matériau, mais il n'existe en revanche aucun travail fournissant des indications sur le comportement à moyen et long terme de ces réhabilitations.

Pour notre cas, l'armature zircone était parfaitement indiquée, compte tenu de ses excellentes propriétés mécaniques et pour ses qualités optiques (tableau I). Quant à la céramique de stratification, elle permettra d'assurer un joint céramique périphérique indispensable pour le collage.

Pour des raisons techniques, il n'est pas possible de réaliser des bridges collés tout céramiques selon les mêmes principes que pour les céramo-métalliques. Tant l'épaisseur des ailettes de collage que la dimension du connecteur interproximal doivent être supérieures à celles préconisées pour les bridges à armature métallique (17).

Pour les travaux céramo-métalliques, la préparation de la dent pilier vise à créer une connexion de type glissière, avec un seul degré de liberté et un frottement d'adhérence entre l'intrados de l'armature et la dent pilier. En revanche, pour les travaux tout céramiques, il est nécessaire d'obtenir une assise passive par une préparation adéquate, arrondie. De plus, les systèmes tout céramiques nécessitent, pour des raisons techniques également, une préparation plus invasive par rapport aux systèmes céramométalliques.

WALTER (18) a décrit les principes de préparations pour bridge collé réalise avec le système IPS EMPRESS 2.

I Réalisation de préparation légèrement concaves de 0,5 millimètres d'épaisseur sur les faces palatines des dents bordant l'édentement.

I Respect de la translucidité des bords incisifs et des contacts occlusaux en laissant la limite de préparation à 2 millimètres des bords incisifs.
I Respect des faces proximales réalisant les points de contacts opposés à l'édentement en laissant la préparation à 1 millimètre de ceux-ci.

I Dans la zone édentée les préparations s'arrêtent sous forme d'une rainure bien marquée en arrière du point de contact.

1 Un congé de $0,8 \mathrm{~mm}$ de profondeur est réalisé en cervical, dans l'émail supra-gingival.

La future prothèse doit respecter les critères suivants : I Les zones de jonction doivent présenter des sections de 12 millimètres carrés.

\L'épaisseur des ailettes doit être en moyenne d'1 millimètre.

L'utilisation des vitrocéramique (disilicate de lithium) permet optimiser encore davantage le collage de ces bridges collés. WALTER précise que cette « solution ne pourra être généralisée qu'après la parution de résultats à long terme ».

HEYMANN (19) décrit lui aussi un bridge uniquement composé de céramique: le CAROLINA Bridge. La différence essentielle consiste dans le fait qu'il ne prépare pas les faces palatines des piliers dentaires, il, réalise seulement une surface rugueuse sur les faces proximales qui correspondra à la future zone de collage. Il indique que cette solution est plutôt destinée à une utilisation sur un court terme, en temporisation avant une prothèse implanto-portée.

Kern en 2005 (11) préconise l'utilisation de l'InCeram Zirconia ${ }^{\oplus}$. L'armature peut être usinée à l'aide d'un procédé de CFAO. La réduction amélaire est de 0,5 à $0,7 \mathrm{~mm}$ minimum et la préparation comprend des boîtes proximales de $2 \mathrm{~mm} \times 1 \mathrm{~mm}$ x $0,5 \mathrm{~mm}$.

La zircone ne peut pas être mordancée par les acides courants utilisés en technique dentaire. Une adhérence cliniquement suffisante à la zircone peut être atteinte par l'utilisation d'un adhésif contenant des monomères de phosphate (p. ex. Alloy- Primer ou Clearfil ${ }^{\text {m }}$ Ceramic Primer [Kuraray], Monobond ${ }^{\circ}$ Plus [Ivoclar Vivadent]) ou par des composites contenant des monomères de phosphate (p. ex. la famille Panavia ${ }^{\mathrm{m}}$ [Kuraray], Rely ${ }^{\text {mit }}$ Unicem [3M Espe]), en combinaison avec un traitement préalable par un sablage à l'oxyde d'aluminium standardisé appliqué à faible pression (Kern \& Wegner 1998, Kern et coll. 2009, Yang et coll. 2010) $(20,21,22)$.

Enfin pour ce qui la configuration du bridge, Mathias Kern en 1997 (10), décrit les bridges Cantilever tout céramique à un seul pilier qui permettent une mobilité physiologique. Son étude montre à 10 ans un taux de survie de $73,9 \%$ avec deux piliers et $92,3 \%$ avec un seul pilier (11). La pertinence repose sur l'obtention d'un plus grand degré de mouvement de la dent support. 
Parmi les avantages des bridges à une ailette, il y a lieu d'évoquer la préparation ménageant encore davantage les tissus dentaires, la fabrication plus rationnelle et la possibilité de détecter immédiatement une éventuelle perte de rétention. Du point de vue parodontal, le design à une ailette facilite l'hygiène buccodentaire du patient. De plus, la réduction du design comportant dès lors un seul connecteur a des avantages du point de vue esthétique et permet de réaliser des travaux comprenant un diastème. Cependant dans ces études, les bridges cantilevers sont souvent sélectionnés dans des conditions cliniques favorables avec de faibles contraintes occlusales sur l'élément en extension. Les dents supports sont généralement les canines pour remplacer les latérales, les centrales pour les latérales et parfois centrale pour centrale. Ceci ne peut bien sur pas s'appliquer à notre situation clinique.

Enfin, bien que les premières études cliniques de longue durée ont montre résultats prometteurs (Kern 2005, Holt \& Drake 2008) $(11,23)$, Ries et coll. 2006 $(12,13)$ il faut signaler qu'aucune revue de littérature n'est disponible sur la survie à long terme des bridges collés à infrastructure de céramique et que les preuves de leur pérennité clinique font encore défaut à ce jour.

\section{CONCLUSION}

La zircone, matériau promis à un grand avenir et déjà couronné de nombreux succès, s'est imposée en odontologie pour pallier l'utilisation de l'armature métallique. Ses propriétés, découvertes progressivement au fur et à mesure de son utilisation, nous ont permis de repousser nos limites, Le bridge collé en zircone constitue un bel exemple. Il peut se substitue au traitement implantaire dans des indications bien précises. Cependant, un certain nombre de questions cliniques, telles que l'épaisseur nécessaire du matériau, la résistance aux fractures, le conditionnement optimal de la céramique et la fiabilité à long terme.

Ainsi les bridges collés tout céramiques ne sont pas encore recommandés d'une façon générale, mais plutôt en tant qu'alternative thérapeutique dans des cas sélectionnés.

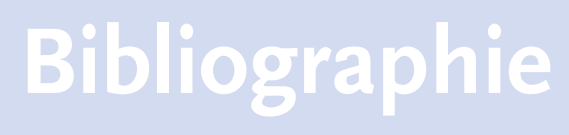

[1] Dietschi D, Dietschi JM, Schatz JP. Remplacement des dents dans le secteur esthétique : modalités conservatrices et prothétiques adhésives. Réalités cliniques vol. 10 nº 11999 pp. 27-41.

[2] Behr M, Leibrock A, Stich W, Rammelsberg P, Rosentritt M, et Handel G. Adhesive-fixed partial dentures in anterior and posterior areas : results of an on going prospective study begun in 1985 . Clin Oral Invest 19982 : 21-35.

[3] Hanson O, Moberg LE. Clinical evaluation of resin-bonded prostheses. Int J Prosthodont 19925 : 533-541.

[4] Samama Y. Fixed bonded prosthodontics: a 10-year follow-up report. Part II: clinical assesemnts. Int J Periodont Rest Dent 199616 : 53- 59.

[5] Rochette AL. Attachment of a splint to enamel of lower anterior teeth. J Prosthet Dent 1973 Oct;30(4):418-423.

[6] Thompson VP, Del Castillo E, Livaditis GJ. Resin-bonded retainers. Part I: Resin bond to electrolytically etched nonprecious alloys. J Prosthet Dent 1983 Dec;50(6):771-779.
[7] Blatz MB. Bonding to cast-metal and porcelainfused-to-metal restorations. Pract Proced Aesthet Dent 2002 14: 294.

[8] Uusalo EK, Lassila VP, Yli-Urpo AU. Bonding of dental porcelain to ceramic-metal alloys. J Prosthet Dent 1987 57: 26-29.

[9] Kern M, Knode H, Strub JR. The allporcelain, resin-bonded bridge. Quintessence Int 1991 22: 257-262.

[10] Kern M, Gläser R. Cantilevered allceramic, resinbonded fixed partial dentures: a new treatment modality. J Esthet Dent 1997 9: 255-264.

[11] Kern M. Clinical long-term survival of two-retainer and single-retainer allceramic resinbonded fixed partial dentures. Quintessence Int 2005 36: 141-147.

[12] Ries S, Wolz J, Richter E. Effect of design of allceramic resin-bonded fixed partial dentures on clinical survival rate. Int J Periodontics Restorative Dent 2006 26: 143-149.

[13] Holt LR, Drake B. The Procera Maryland Bridge: a case report. J Esthet Restor Dent 2008 20: $165-173$.

[14] Duarte SJ, Phark J, Tada T, Sadan A. Resin-bonded fixed partial dentures with a new modified zirconia surface: a clinical report. $J$ Prosthet Dent 2009 102: 68-73.
[15] Edelhoff D, Speikermann H, Rübben A, Yildirim M. Kronen-und Brückengerüste aus hochfester Presskeramik. Quintessenz 199950 : 177-189.

[16] Kern M, Strub JR. Klinische Anwendung und Bewährung von Adhäsivbrücken aus der Aluminiumoxidkeramik In-Ceram. Dtsch Zahnärtzl Z 199247 : 532-539.

[17] Christian Bühler-Freyl Carlo P. Marinello1 Quelle est la place des pontsadhésifs métallo-céramiques à l'époque du tout céramique et de l'implantologie ? Rev Mens Suisse Odontostomatol 2011 Vol. 121.

[18] Walter B. Traitements des agénésies dentaires par bridges tout céramiques. $\mathrm{Cah}$ Prothèse 2003 ; 121 : 7-19.

[19] Heymann HO. The Carolina bridge: a novel interim all porcelain bonded ridge. $J$ Esthet Restor Dent 2006 ; 18 (2) 81-92.

[20] Kern M, Wegner SM. Bonding to zirconia ceramic: adhesion methods and their durability. Dent Mater 199814 : 64-71.

[21] Kern M, Barloi A, Yang B. Surface conditioning influences zirconia ceramic bonding. J Dent Res 200988 : 817-822.

[22] Yang B, Barloi A, Kern M. Influence of air-abrasion on zirconia ceramic bonding using an adhesive composite resin. Dent Mater 2010 26: $44-50$. 